\title{
Efficacy of tolvaptan for fluid management after cardiovascular surgery: A systematic review and meta-analysis of randomized control trials
}

\author{
HUILING CHEN $^{1}$, WEIHONG JIANG ${ }^{1}$, XIAOGANG LI $^{1}$, ZHAOHUI MENG $^{2}$, \\ HUAMEI CHEN $^{2}$, JIAYING LI ${ }^{1}$, JUNWEN WANG ${ }^{1}$ and XIEXIONG ZHAO ${ }^{1}$ \\ ${ }^{1}$ Department of Cardiology, the Third Xiangya Hospital of Central South University, Changsha, Hunan 410013; \\ ${ }^{2}$ Department of Cardiology, the First Affiliated Hospital of Kunming Medical University, Kunming, Yunnan 660034, P.R. China
}

Received November 18, 2019; Accepted January 9, 2020

DOI: $10.3892 /$ etm.2020.9007

\begin{abstract}
The purpose of this study was to systematically search the literature and analyze evidence from randomized controlled trials (RCTs) comparing tolvaptan with conventional diuretics for postoperative fluid management in cardiac surgery patients. An electronic search of PubMed, Scopus, BioMed Central, CENTRAL (Cochrane Central Register of Controlled Trials) and Google scholar databases was carried out up to 1st December 2019. Four RCTs were included. Tolvaptan was co-administered with conventional diuretics in all the studies. The mean postoperative urine output was significantly greater in patients receiving tolvaptan as compared to controls $(\mathrm{MD}=0.39 ; 95 \% \mathrm{CI}$ : 0.17 to $0.61 ; \mathrm{P}=0.006, \mathrm{I}^{2}=48 \%$ ). Body weight of patients on tolvaptan returned to pre-operative levels significantly earlier ( $\mathrm{MD}=-1.57 ; 95 \% \mathrm{CI}:-2.48$ to $-0.66 ; \mathrm{P}=0.007, \mathrm{I}^{2}=50 \%$ ). There was statistical significant difference in the highest postoperative serum sodium levels $(\mathrm{MD}=2.34$; $95 \% \mathrm{CI}$ : -1.65 to 3.03 ; $\left.\mathrm{p}<0.00001, \mathrm{I}^{2}=0 \%\right)$, lowest serum sodium levels $(\mathrm{MD}=2.05$; 95\% CI: 1.41 to $2.68 ; \mathrm{p}<0.00001, \mathrm{I}^{2}=0 \%$ ) and mean serum sodium levels $(\mathrm{MD}=1.69 ; 95 \% \mathrm{CI}$ : 0.98 to $2.40 ; \mathrm{p}<0.00001$, $\mathrm{I}^{2}=0 \%$ ) between the tolvaptan and control groups. Lowest serum potassium was significantly higher with tolvaptan as compared to the control group $(\mathrm{MD}=0.10 ; 95 \% \mathrm{CI}: 0.01$ to $0.18 ; \mathrm{P}=0.03, \mathrm{I}^{2}=19 \%$ ). There was no significant difference in the length of ICU stay or incidence of arrhythmias between the two groups. The quality of the included studies was not high. Within the limitations of our study, our results indicate that co-administration of tolvaptan with low dose of conventional diuretics significantly increases urine output while
\end{abstract}

Correspondence to: Professor Weihong Jiang, Department of Cardiology, the Third Xiangya Hospital of Central South University, 138 Tongzipo Road, Changsha, Hunan 410013, P.R. China E-mail: jwhxy1212@163.com

Key words: tolvaptan, vasopressin antagonist, cardiac surgery, fluid management maintaining electrolyte balance in postoperative cardiac surgery patients. Faster return of body weight to pre-operative levels is evident with tolvaptan. Further high-quality RCTs are required to confirm this evidence.

\section{Introduction}

Appropriate control of body fluids is extremely important while managing postoperative cardiac surgery patients. Any cardiac procedure involving or not involving cardiopulmonary bypass can cause a significant increase in total body water due to high fluid load (1). The surgical stress of the procedure and the use of extra-corporeal circulation also contribute to increased body water levels (2). Management of such patients has been primarily dependent on the use of loop diuretics such as furosemide, which is the drug of choice. However, continual use of loop diuretics is associated with several adverse effects including electrolyte disturbances, deterioration of kidney function and diuretic resistance (3). Furosemide acts on the thick ascending loop of Henle where it inhibits sodium reabsorption thereby leading to hyponatremia. Hyponatremia, in turn, reduces drug efficacy and an increased dose of furosemide is required for maintaining diuresis. Higher doses further aggravate hyponatremia, leading to an endless cycle (4). To overcome these limitations of loop diuretics, newer therapeutic agents have been developed.

Tolvaptan is an orally administered diuretic that selectively antagonizes vasopressin V2 receptors. The unique property of the drug is that it causes electrolyte-free diuresis (5). In a randomized placebo-controlled crossover trial of heart failure patients, Costello-Boerrigter et al (5) demonstrated the similar diuretic efficacy of tolvaptan and furosemide. However, unlike furosemide, tolvaptan was not associated with electrolyte imbalance and did not affect renal function. Due to its beneficial pharmacological properties, tolvaptan has been approved for managing heart failure patients with numerous studies establishing its efficacy and safety profile (6). Over the last decade, researchers have also evaluated the efficacy of tolvaptan for postoperative fluid management in cardiac surgery (1,7). In one of the earliest studies, Nishi et al (1) compared a prospective cohort of 64 heart valve patients managed using tolvaptan with 
a historic control group of 55 patients managed with conventional diuretics. The authors of that study found tolvaptan to be an effective diuretic without causing electrolyte disbalance or worsening of renal function. Other published retrospective observational studies have also reported similar results (7-9). However, a significant limitation of observational studies is the high risk of bias. The best possible evidence to guide clinical practice has always been in the form of a meta-analysis of randomized controlled trials (RCTs). Therefore, the aim of the present study was to systematically search the literature and analyze evidence from RCTs comparing tolvaptan with conventional diuretics for postoperative fluid management in cardiac surgery patients.

\section{Materials and methods}

Study selection and search strategy. We conducted this systematic review and meta-analysis following the guidelines of the PRISMA statement (Preferred Reporting Items for Systematic Reviews and Meta-analyses) (10) and the Cochrane Handbook for Systematic Reviews of Intervention (11). We followed the common medicine PICO (Population, Intervention, Comparison, Outcome) framework for selecting studies. This review included only randomized controlled trials (RCTs) conducted on adult patients undergoing any type of cardiac surgery (Population), and compared the efficacy (Outcomes) of tolvaptan (Intervention) with conventional diuretics (Comparison). Studies evaluating the use of tolvaptan without any cardiac surgical intervention, on the pediatric population and patients with kidney diseases were excluded. We also excluded non-randomized studies, retrospective observational studies, and case series.

An electronic literature search without any language or time restriction was carried out on PubMed, Scopus, BioMed Central, CENTRAL (Cochrane Central Register of Controlled Trials) and Google scholar databases up to 1st December 2019. Keywords 'Tolvaptan', 'Vasopressin receptor antagonist', 'surgery', 'cardiac surgery', 'diuretics', and 'randomised controlled trials' were used in different combinations by two independent reviewers. Reference lists of eligible studies and pertinent review articles were hand-searched for the identification of any other studies.

Data extraction and outcomes. Search results were first screened by careful evaluation of titles and abstracts. Full-texts of selected trials were then obtained for further evaluation. Any discrepancies between the two reviewers were resolved by discussion. Using a standardized data collection sheet, data were extracted from the included trials by the two reviewers independently. We extracted names of study authors, year of the study, sample size, inclusion/exclusion criteria, demographic details of the sample, tolvaptan protocol, use of other diuretics and study outcomes. Corresponding authors were contacted for any missing data via emails.

The primary outcomes of interest were postoperative urine output and the number of days for the return to pre-operative body weight. Other secondary outcomes were serum levels of sodium and potassium, post-operative kidney function, length of ICU (intensive care unit) stay and new postoperative arrhythmias.
Risk of bias. Since the review included only RCTs, we utilized the Cochrane Collaboration risk assessment tool for quality assessment of the included trials (12). We rated studies for risk of bias on the following items: random sequence generation, allocation concealment, blinding of participants and personnel, blinding of outcome assessment, incomplete outcome data, selective reporting, and other biases. The risk of bias was presented as a summary chart with a green circle denoting low risk of bias, yellow circle denoting unclear risk of bias and red circle denoting a high risk of bias for the particular item.

Statistical analysis. Mean \pm standard deviation (SD) values were extracted for continuous variables and the number of events for categorical variables. Studies in which continuous data were presented only in graphical format, Engauge Digitizer Version 12.1 was used to extract numerical values from study graphs by two independent reviewers. Considering the heterogeneity among studies, we used the random-effects model to pool data. Continuous variables were pooled using mean difference (MD) and 95\% confidence interval (CI) while categorical data were summarised using the Mantel-Haenszel odds ratio (OR) and 95\% CI. Meta-analysis was conducted only if at least three studies reported data on the same scale. We assessed inter-study heterogeneity using the $\mathrm{I}^{2}$ statistic. Values of $25-50 \%$ represented low, $50-75 \%$ medium and $>75 \%$ represented substantial heterogeneity. The software Review Manager (RevMan, version 5.3; Nordic Cochrane Centre [Cochrane Collaboration], Copenhagen, Denmark; 2014) was used for conducting the statistical analysis. Since the number of studies was less than 10 , publication bias was not assessed.

\section{Results}

The results of the literature search and selection process are denoted in Fig. 1. A total of 11 studies were screened by their full text. Five were excluded as they were not RCTs $(1,7,9,13,14)$. One study was conducted on pediatric patients (15), and another on patients with chronic kidney disease (8). Finally, four studies were included in this systematic review and meta-analysis $(2,16-18)$. Details of the included studies are presented in Table I. All four trials were conducted in Japan and published between January 2016 and February 2018. Patients with chronic renal disease were excluded from all the studies. The number of patients randomized to the tolvaptan group varied from 19 patients in one study to 147 patients in another trial. The mean age of included patients was more than 65 years in all the studies. The dose of tolvaptan was standard at $7.5 \mathrm{mg}$ across trials but the duration of therapy varied. Tolvaptan was given as needed after 3 days, for a total of 5 days or until the body weight returned to pre-operative levels. Conventional diuretics were administered in both tolvaptan and control groups in all the studies. One trial studied tolvaptan in two separate groups of high dose $(15 \mathrm{mg})$ and low dose (7.5 mg) (18). Data from the low dose group were extracted for this review.

Primary outcomes. Three trials reported data on urine output $(2,17,18)$ and number of days to return to pre-operative 


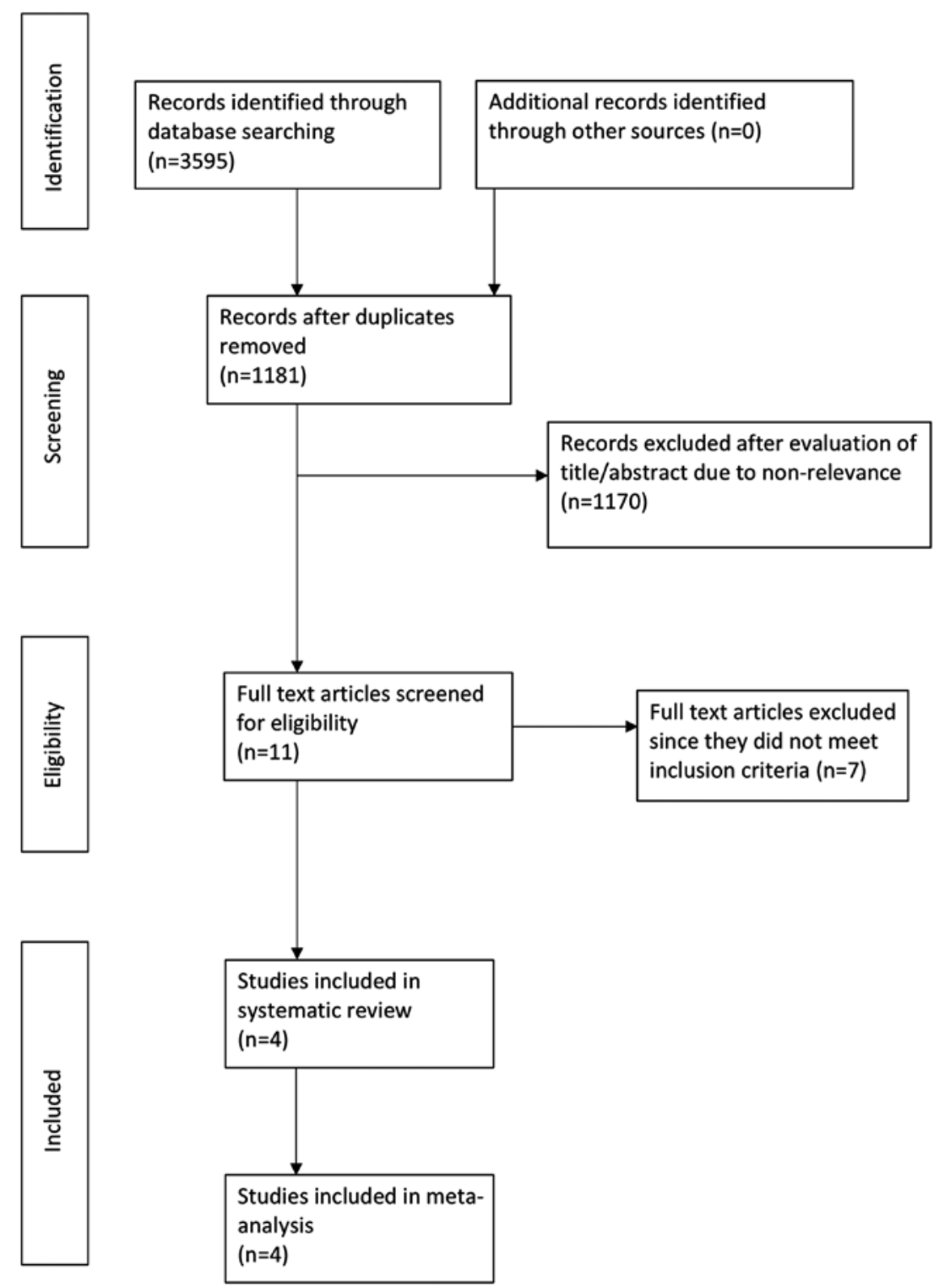

Figure 1. Study flow chart.

body weight $(2,16,18)$ as mean \pm SD. Mean post-operative urine output was significantly greater in patients receiving tolvaptan as compared to controls $(\mathrm{MD}=0.39 ; 95 \% \mathrm{CI}: 0.17$ to $0.61 ; \mathrm{P}=0.0006, \mathrm{I}^{2}=48 \%$ ) (Fig. 2). Similarly, the body weight of patients on tolvaptan returned to pre-operative levels significantly earlier as compared to control group $(\mathrm{MD}=-1.57$; 95\% CI: -2.48 to $-0.66 ; \mathrm{P}=0.0007, \mathrm{I}^{2}=50 \%$ ) (Fig. 3 ).

Secondary outcomes. Data on highest, lowest and mean serum sodium levels were available from three studies and were pooled separately $(2,17,18)$. There was a statistically significant difference in the highest postoperative serum sodium levels $\left(\mathrm{MD}=2.34 ; 95 \% \mathrm{CI}:-1.65\right.$ to 3.03; $\left.\mathrm{p}<0.00001, \mathrm{I}^{2}=0 \%\right)$ (Fig. 4A) and lowest post-operative serum sodium levels $(\mathrm{MD}=2.05$; 95\% CI: 1.41 to 2.68 ; $<<0.00001, \mathrm{I}^{2}=0 \%$ ) (Fig. $4 \mathrm{~B}$ ) between the tolvaptan and the control groups. Similarly, the mean postoperative serum sodium levels were significantly higher in the tolvaptan group as compared to the controls $(\mathrm{MD}=1.69$; 95\% CI: 0.98 to $2.40 ; \mathrm{p}<0.00001, \mathrm{I}^{2}=0 \%$ ) (Fig. 4C). The study not included in the meta-analysis also reported significantly higher levels of serum sodium from postoperative day 3 to 6 in patients receiving tolvaptan (16).

For serum potassium levels, data only on the lowest serum potassium level were available for a meta-analysis. Lowest serum potassium was significantly higher with tolvaptan as compared to control (MD=0.10; $95 \% \mathrm{CI}$ : 0.01 to $0.18 ; \mathrm{P}=0.03$, $\mathrm{I}^{2}=19 \%$ ) (Fig. 5). Descriptive analysis of results from three studies indicated no significant difference in mean serum potassium levels between the two study groups $(2,16,17)$.

There was no difference in the length of ICU stay (MD=-0.09; 95\% CI: -0.44 to $0.26 ; \mathrm{P}=43, \mathrm{I}^{2}=60 \%$ ) (Fig. 6 ) and the number of new post-operative arrhythmias between the two groups $\left(\mathrm{OR}=0.92 ; 95 \% \mathrm{CI}: 0.43\right.$ to $\left.1.97 ; \mathrm{P}=0.84, \mathrm{I}^{2}=0 \%\right)$ (Fig. 7).

Kidney function. In the absence of coherent data for a meta-analysis, kidney function outcomes from included studies are presented descriptively. Kato et al (17) measured blood urea nitrogen (BUN) and fractional excretion of urea nitrogen (FEUN) in both groups up to postoperative 


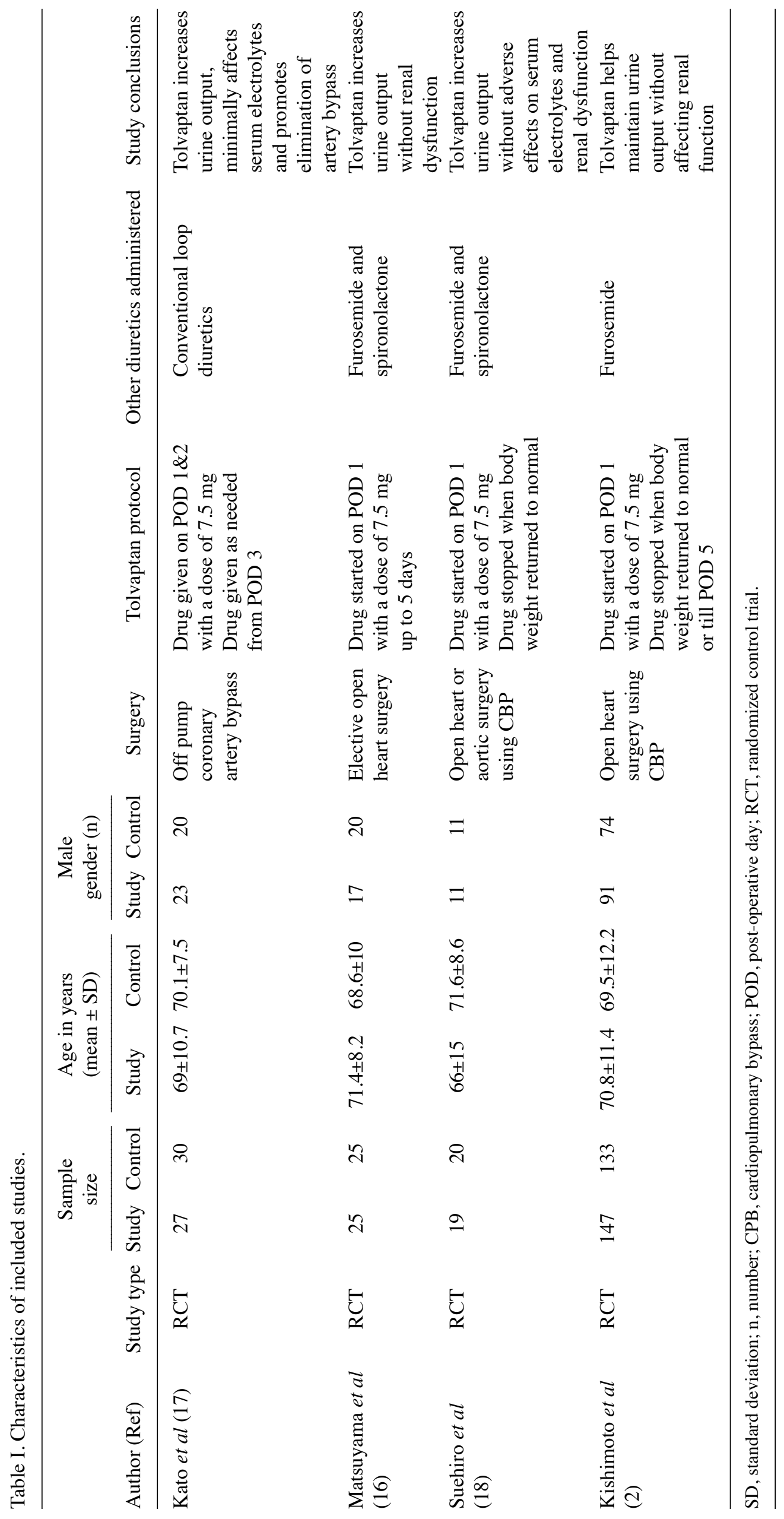




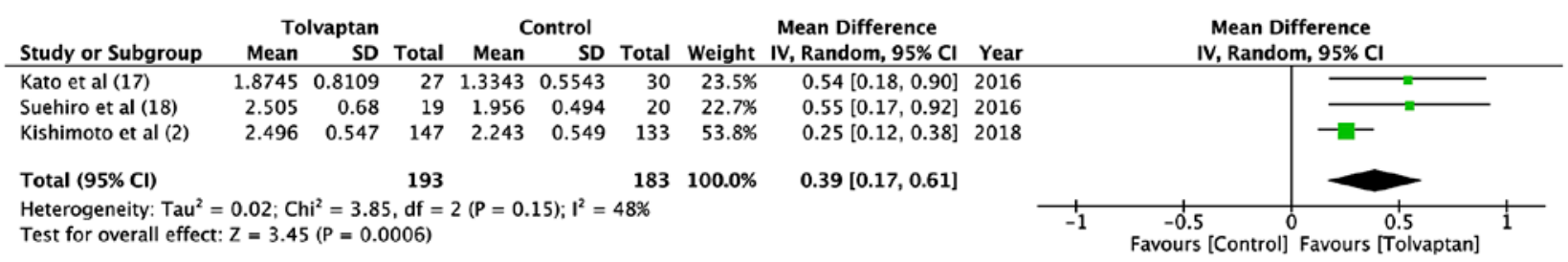

Figure 2. Forrest plot for urine output.

\begin{tabular}{|c|c|c|c|c|c|c|c|c|c|c|c|c|c|}
\hline \multirow[b]{2}{*}{ Study or Subgroup } & \multicolumn{3}{|c|}{ Tolvaptan } & \multicolumn{3}{|c|}{ Control } & \multicolumn{3}{|c|}{ Mean Difference } & \multirow{2}{*}{\multicolumn{4}{|c|}{$\begin{array}{c}\text { Mean Difference } \\
\text { IV, Random, } 95 \% \mathrm{Cl}\end{array}$}} \\
\hline & Mean & SD & Total & Mean & SD & Total & Weight & IV, Random, 95\% Cl & Year & & & & \\
\hline Matsuyama et al (16) & 4.7 & 1.6 & 25 & 6.8 & 1.9 & 25 & $38.9 \%$ & $-2.10[-3.07,-1.13]$ & 2016 & & -1 & & \\
\hline Suehiro et al (18) & 6.7 & 5.5 & 19 & 9.5 & 5.6 & 20 & $6.2 \%$ & $-2.80[-6.28,0.68]$ & 2016 & & & . & \\
\hline Kishimoto et al (2) & 3.97 & 1.95 & 147 & 5.02 & 2.83 & 133 & $54.9 \%$ & $-1.05[-1.63,-0.47]$ & 2018 & & 를 & & \\
\hline Total $(95 \% \mathrm{CI})$ & & & 191 & & & 178 & $100.0 \%$ & $-1.57[-2.48,-0.66]$ & & & & & \\
\hline $\begin{array}{l}\text { Heterogeneity: } \mathrm{Tau}^{2}= \\
\text { Test for overall effect: }\end{array}$ & $\begin{array}{l}0.31 ; C l \\
Z=3.3\end{array}$ & $h i^{2}=3$ & $\begin{array}{l}.99, \mathrm{df} \\
0.0007\end{array}$ & $=2$ & 0.14 & $; 1^{2}=$ & $50 \%$ & & & -10 & $\begin{array}{l}-5 \\
-5\end{array}$ & Favours IC & $\begin{array}{l}5 \\
5 \\
\text { Control] }\end{array}$ \\
\hline
\end{tabular}

Figure 3. Forrest plot for days for body weight to return to pre-operative levels.

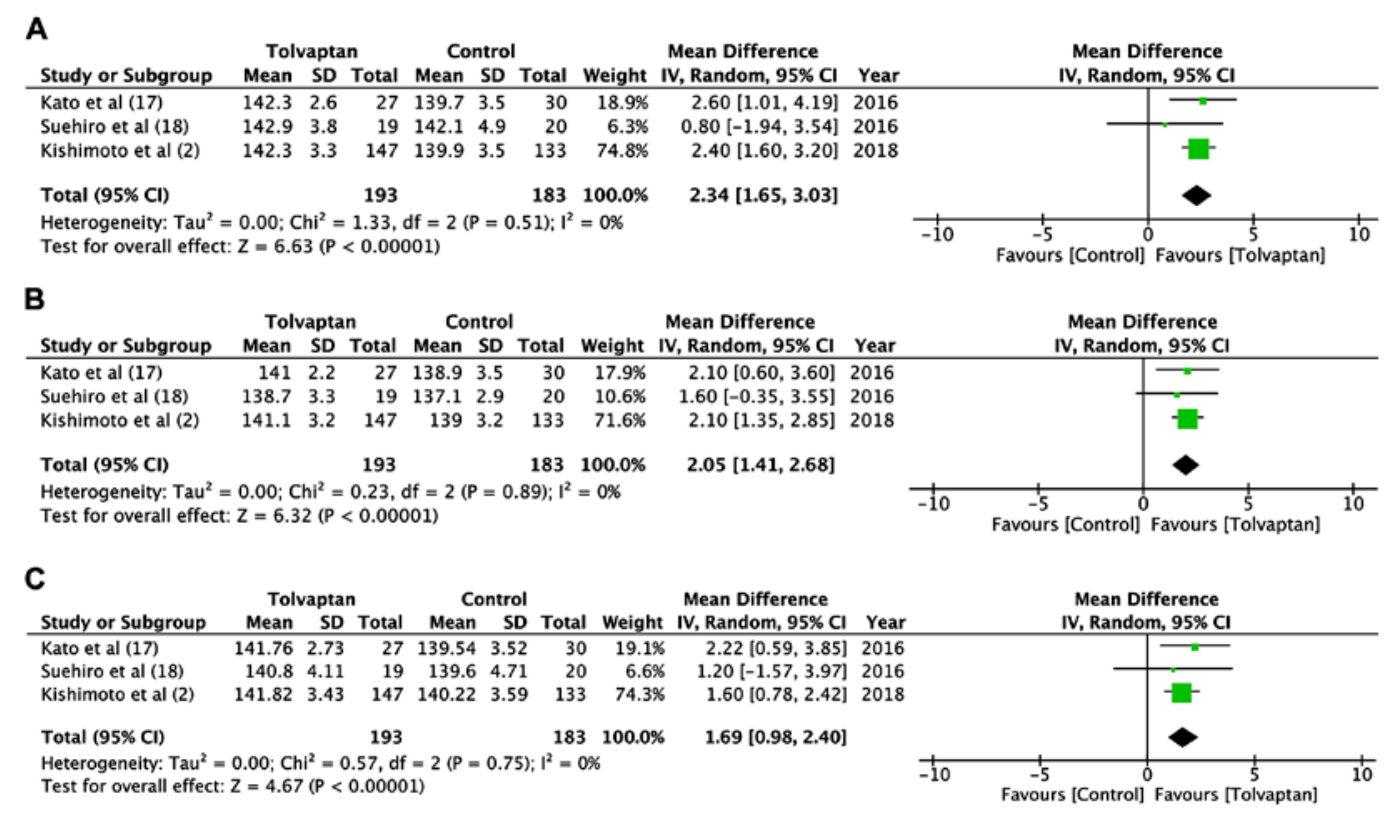

Figure 4. Forrest plot for (A) Highest serum sodium level. (B) Lowest serum sodium level. (C) mean serum sodium level.

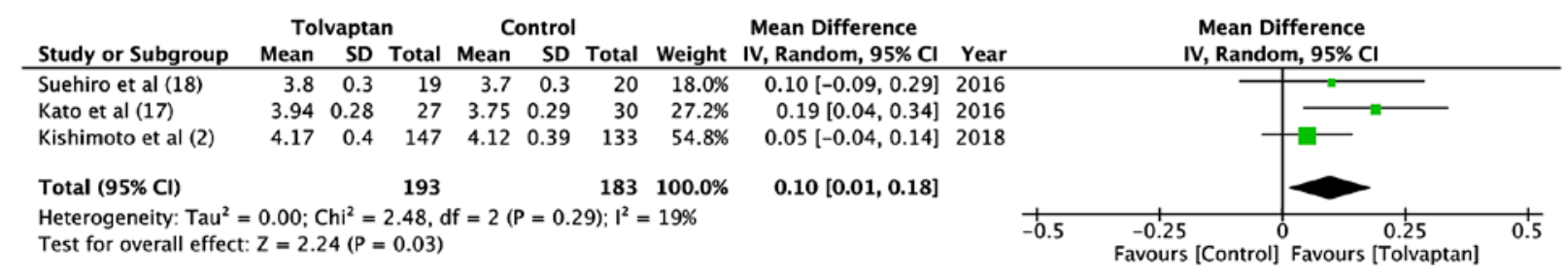

Figure 5. Forrest plot for lowest serum potassium level.

day 7. There was no statistical significant difference in BUN between the two groups; however, FEUN was significantly higher on postoperative day 2 and 5 in the tolvaptan group. In the study of Matsuyama et al (16), no significant difference was evident in serum creatinine and BUN levels between the two groups except on postoperative day 3 when the levels were significantly lower in the tolvaptan group. Suehiro et al (18) found no significant difference in the highest serum creatinine levels between the two study groups. No other kidney function data was recorded in their trial. Kishimoto et al (2) evaluated the incidence of worsening of renal function which was defined as an increase in the serum creatinine concentration of $\geq 0.3 \mathrm{mg} / \mathrm{dl}$ compared to the preoperative value at any given day until postopera- 


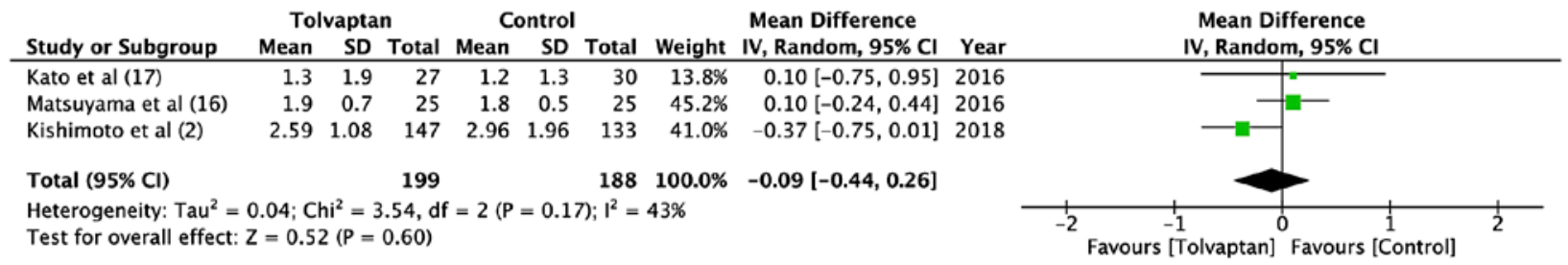

Figure 6. Forrest plot for length of ICU stay.

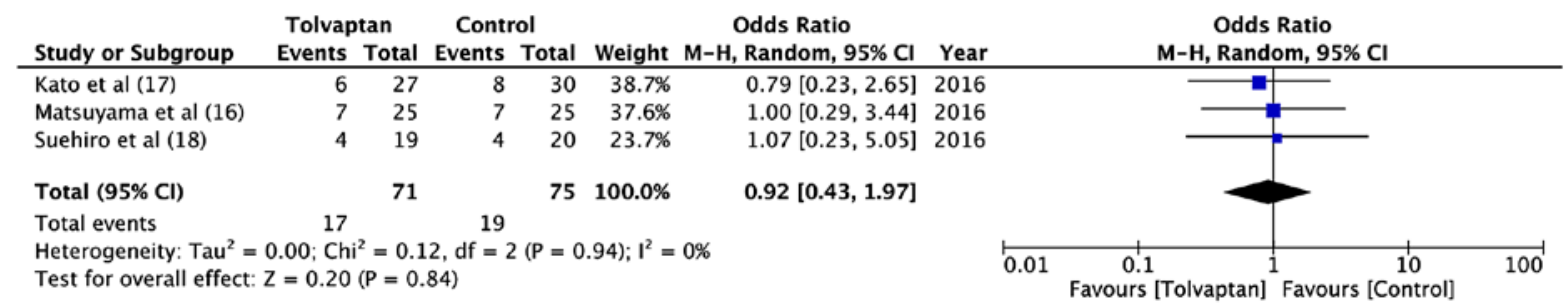

Figure 7. Forrest plot for incidence of arrhythmia.

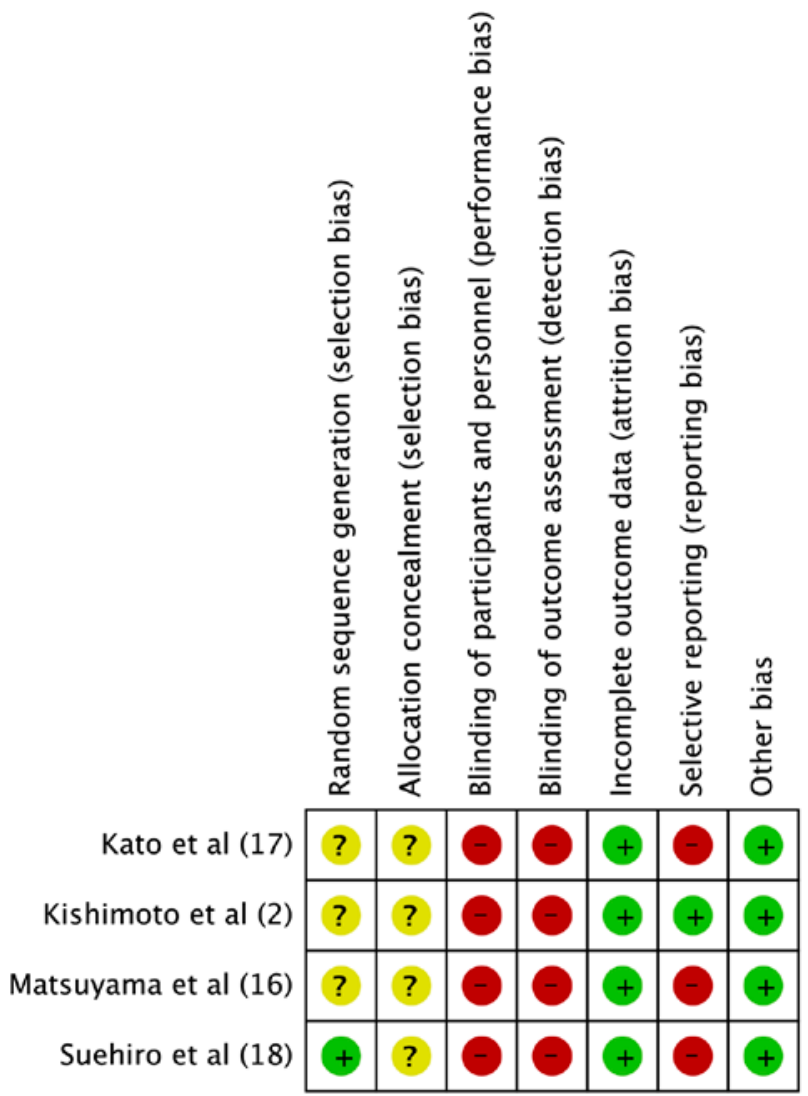

Figure 8 . The risk of bias summary of included studies.

tive day 5 . The incidence of worsening renal function was significantly less in the tolvaptan group as compared to the control group.

Risk of bias analysis. Fig. 8 presents the authors' judgment of the risk of bias summary of included studies. The randomization technique was clearly described by only one study (18). None of the trials were blinded. Selective reporting was observed in all except one trial (2).

\section{Discussion}

Patients undergoing cardiac surgery experience significant operative stress which leads to increased secretion of arginine vasopressin (AVP) (19). High levels of AVP act on the renal collecting duct causing excessive water reabsorption and subsequent water imbalance in the early postoperative period. Findings have shown that post-operative cardiac patients tend to have a higher level of serum AVP concentration as compared to non-cardiac surgery patients (20). Consequently, in view of increased water imbalance in this cohort, the appropriate and timely use of diuretics is extremely crucial to prevent morbidity and mortality. The purpose of this study was therefore to investigate the efficacy of tolvaptan, an aquaretic diuretic, which acts by selectively inhibiting AVP from binding to V2 receptors in the kidney and its role in cardiac surgery patients.

After a thorough literature search, a total of four RCTs were found to compare tolvaptan with loop diuretics for cardiac surgery patients. Importantly, patients randomized to tolvaptan were also given low doses of loop diuretics and therefore the results of this meta-analysis do not represent outcomes of singular tolvaptan administration. The primary outcome of our analysis was a significantly higher urine output and a lower number of days required to return to pre-operative body weight with tolvaptan. Our results concur with findings of studies on congestive heart failure (CHF) patients which also demonstrated higher urine output and a decrease in body weight when tolvaptan was added to standard diuretic therapy (21-23). However, although body weight tends to return to pre-operative levels after the 5 th postoperative day (24), excessive fluid retention in the early period can cause a worsening of respiratory function due to pulmonary congestion. This, in turn, increases the duration of oxygenation and bed rest (2). It is postulated that higher urine output and faster return to pre-operative body weight with tolvaptan may contribute to faster patient ambulation as well as reduced ICU and hospital stay $(2,25)$. However, in our analysis, we did not find any difference in ICU stay between the two study groups. 
Length of ICU stay could have been influenced by several other factors such as the type of surgical procedure, duration of surgery, and patient response to treatment.

An important secondary outcome of our study was postoperative electrolyte levels. Loop diuretics, not only cause hyponatremia, but they also increase sodium transport to the distal tubule which stimulates the aldosterone-sensitive sodium pump, leading to potassium excretion and consequent hypokalaemia (4). Tolvaptan, in contrast to loop diuretics, is expected to maintain electrolyte levels with a water-only diuresis. Data from the included studies did not permit a per-day analysis of sodium and potassium levels and meta-analysis only for highest, lowest and mean sodium and lowest potassium levels were carried out in this study. Our results indicate that the highest, lowest, as well as mean sodium levels, were significantly higher in the tolvaptan group as compared to patients receiving only loop diuretics. The lower dose of loop diuretics and the use of tolvaptan probably resulted in higher sodium levels in the study group. Although hyponatremia is an independent predictor of postoperative complications after cardiac surgery (26), it is important to note that the lowest sodium levels in the control group were not below $135 \mathrm{mEq} / \mathrm{l}$. Secondly, electrolyte-free diuresis with tolvaptan can lead to hypernatremia, which is a major side-effect (27). However, none of the four studies reported extreme hypernatremia with tolvaptan. The exception was Kishimoto et al (2) who reported sodium levels of $>147 \mathrm{mEq} / 1$ in 5 patients with tolvaptan therapy, which returned to normal after discontinuation of the drug.

As with sodium, maintenance of serum potassium levels is also crucial in cardiac surgery patients. Significant hypokalaemia can lead to supraventricular and ventricular arrhythmias, which can increase morbidity, prolong hospital stay, and escalate healthcare expenses for a cardiac patient (28). Our results indicated that the lowest potassium levels were significantly higher with tolvaptan as compared to the control group, albeit with small effect size and the lower end of the CI close to zero (95\% CI: 0.01-0.18). In addition, we found no difference in the incidence of arrhythmias between the two cohorts. The descriptive analysis revealed there was no difference in the mean potassium levels between the tolvaptan and control groups in any of the included studies. Absence of any effect of tolvaptan on potassium excretion and use of potassium-sparing diuretics along with furosemide may have prevented significant hypokalaemia in both groups.

An aggressive diuretic treatment has been associated with deterioration of renal function. Loop diuretics are known to inhibit sodium-chloride transport in the ascending Henle loop and increase flow in the distal nephron. This triggers tubulo-glumerular feedback causing constriction of the afferent arteriole and reduction in glomerular filtration rate (GFR) (29). On the other hand, tolvaptan has no influence on renal blood flow or GFR even in individuals with chronic kidney disease (30). One of the studies excluded from this review has demonstrated tolvaptan to be safe in chronic renal patients undergoing cardiac surgery (8). Similarly, studies on $\mathrm{CHF}$ patients have shown that tolvaptan prevents acute renal failure and reduces the amount of loop diuretic required for the management of such patients (31). The absence of coherent study variables and lack of data precluded a meta-analysis of kidney function in the current study. Three of the four included trials did not report any significant difference in serum creatinine and BUN levels between the two groups while only one trial (2) reported increased incidence of worsening of renal function in the control group. In the absence of sufficient high-quality studies, further trials are required to establish the reno-protective effect of tolvaptan in cardiac surgery patients.

The results of the present study should be interpreted with the following limitations. Firstly, only four RCTs were available for analysis for this review. The majority of the included RCTs had a small sample size. Secondly, the quality of the included trials was not high, which potentially downgrades the level of evidence from our results. Thirdly, there was methodological heterogeneity in the included studies for the surgical procedure, duration of tolvaptan therapy, and perioperative fluid management. Lastly, a meta-analysis on renal function outcomes could not be conducted due to a lack of data.

Nevertheless, to the best of our knowledge, our study is the first meta-analysis of only RCTs evaluating the efficacy of tolvaptan for cardiac surgery patients. The last meta-analysis on this topic was a pooled analysis of RCTs as well as observational studies (25). It omitted one of the four RCTs included in this review while including observational studies on pediatric patients and renal disease patients. Therefore, we believe the results of the present study provide the most optimal level 1 evidence on the role of tolvaptan in cardiac surgery patients. Within the limitations of the current study, the results indicate that co-administration of tolvaptan with low-dose of conventional diuretics significantly increases urine output while maintaining serum sodium and potassium levels in postoperative cardiac surgery patients. Faster return of body-weight to pre-operative levels is seen with tolvaptan. Further high-quality RCTs are required to provide stronger evidence on this topic.

\section{Acknowledgements}

Not applicable.

\section{Funding}

No funding was received.

\section{Availability of data and materials}

The datasets used and/or analyzed during the current study are available from the corresponding author on reasonable request.

\section{Authors' contributions}

HLC and WJ designed the paper. XL, ZM, HMC, JL, JW and $\mathrm{XZ}$ were involved in literature search and data interpreted. HLC, XL and ZM were responsible for the data analysis. HMC prepared the manuscript. WJ edited the manuscript. All authors have read and approved the final manuscript.

\section{Ethics approval and consent to participate}

Not applicable. 


\section{Patient consent for publication}

Not applicable.

\section{Competing interests}

The authors declare that they have no competing interests.

\section{References}

1. Nishi H, Toda K, Miyagawa S, Yoshikawa Y, Fukushima S, Kawamura M, Yoshioka D, Saito T, Ueno T, Kuratani T, et al Effects of tolvaptan in the early postoperative stage after heart valve surgery: Results of the STAR (Study of Tolvaptan for fluid retention AfteR valve surgery) trial. Surg Today 45: 1542-1551, 2015.

2. Kishimoto Y, Nakamura Y, Harada S, Onohara T, Kishimoto S, Kurashiki T, Fujiwara Y and Nishimura M: Can Tolvaptan Protect Renal Function in the Early Postoperative Period of Cardiac Surgery? - Results of a Single-Center Randomized Controlled Study. Circ J 82: 999-1007, 2018.

3. Felker GM: Loop diuretics in heart failure. Heart Fail Rev 17: 305-311, 2012.

4. Haller C, Salbach P, Katus H and Kübler W: Refractory oedema in congestive heart failure: A contributory role of loop diuretics? J Intern Med 237: 211-214, 1995.

5. Costello-Boerrigter LC, Smith WB, Boerrigter G, Ouyang J, Zimmer CA, Orlandi C and Burnett JC Jr: Vasopressin-2-receptor antagonism augments water excretion without changes in renal hemodynamics or sodium and potassium excretion in human heart failure. Am J Physiol Renal Physiol 290: F273-F278, 2006.

6. Sen J, Chung E and McGill D: Tolvaptan for Heart Failure in Chronic Kidney Disease Patients: A Systematic Review and Meta-Analysis. Heart Lung Circ 27: 928-939, 2018.

7. Ito H, Mizumoto T, Tempaku H, Fujinaga K, Sawada $\mathrm{Y}$ and Shimpo H: Efficacy of Tolvaptan on Fluid Management After Cardiovascular Surgery Using Cardiopulmonary Bypass. J Cardiothorac Vasc Anesth 30: 1471-1478, 2016.

8. Yamada M, Nishi H, Sekiya N, Horikawa K, Takahashi T and Sawa Y: The efficacy of tolvaptan in the perioperative management of chronic kidney disease patients undergoing open-heart surgery. Surg Today 47: 498-505, 2017.

9. Kido T, Nishi H, Toda K, Ueno T, Kuratani T, Sakaki M, Takahashi T and Sawa Y: Predictive factors for responders to tolvaptan in fluid management after cardiovascular surgery. Gen Thorac Cardiovasc Surg 65: 110-116, 2017.

10. Moher D, Liberati A, Tetzlaff J and Altman DG; PRISMA Group: Preferred reporting items for systematic reviews and meta-analyses: The PRISMA statement. PLoS Med 6: e1000097, 2009.

11. Higgins $\mathbf{J}$ and Green S (eds): Cochrane Handbook for Systemic Reviews of Interventions Version 5.1.0. The Cochrane Collaboration, 2011. www.handbook.cochrane.org. Updated March, 2011

12. Higgins J, Altman D and Sterne J; Cochrane Statistical Methods Group and the Cochrane Bias Methods Group: Chapter 8: Assessing risk of bias in included studies. In: Cochrane Handbook for Systemic Reviews of Interventions, Version 5.1.0. The Cochrane Collaboration, 2011. www.handbook.cochrane. org. Accessed May 8, 2014.

13. Noguchi K, Tanaka M, Katayama I, Yamabe T, Yuji D, Oosiro N and Sirouzu M: Efficacy of Tolvaptan in Patients with Volume Overload after Cardiac Surgery. Heart Surg Forum 18 E232-E236, 2015.

14. Kato TS, Ono S, Kajimoto K, Kuwaki K, Yamamoto T and Amano A: Early introduction of tolvaptan after cardiac surgery: A renal sparing strategy in the light of the renal resistive index measured by ultrasound. J Cardiothorac Surg 10: 143, 2015

15. Katayama Y, Ozawa T, Shiono N, Masuhara H, Fujii T and Watanabe Y: Safety and effectiveness of tolvaptan for fluid management after pediatric cardiovascular surgery. Gen Thorac Cardiovasc Surg 65: 622-626, 2017.

16. Matsuyama K, Koizumi N, Nishibe T, Iwasaki T, Iwahasi T, Toguchi K, Takahashi S, Iwahori A, Maruno K and Ogino H: Effects of short-term administration of tolvaptan after open heart surgery. Int J Cardiol 220: 192-195, 2016.
17. Kato TS, Nakamura H, Murata M, Kuroda K, Suzuki H, Yokoyama Y, Shimada A, Matsushita S, Yamamoto T and Amano A: The effect of tolvaptan on renal excretion of electrolytes and urea nitrogen in patients undergoing coronary artery bypass surgery. BMC Cardiovasc Disord 16: 181, 2016.

18. Suehiro Y, Hosono M, Shibata T, Sasaki Y, Hirai H, Nakahira A, Kubota Y, Kaku D and Suehiro S: Efficacy and Safety Evaluation of Tolvaptan on Management of Fluid Balance after Cardiovascular Surgery Using Cardiopulmonary Bypass. Osaka City Med J 62: 111-119, 2016.

19. Woods WG, Forsling ML and Le Quesne LP: Plasma arginine vasopressin levels and arterial pressure during open heart surgery. Br J Surg 76: 29-32, 1989.

20. Jochberger S, Mayr VD, Luckner G, Wenzel V, Ulmer H, Schmid S, Knotzer H, Pajk W, Hasibeder W, Friesenecker B, et al: Serum vasopressin concentrations in critically ill patients. Crit Care Med 34: 293-299, 2006

21. Miyazaki T, Fujiki H, Yamamura Y, Nakamura S and Mori T: Tolvaptan, an orally active vasopressin V(2)-receptor antagonist pharmacology and clinical trials. Cardiovasc Drug Rev 25: 1-13, 2007.

22. Gheorghiade $\mathrm{M}$, Gattis WA, O'Connor CM, Adams KF Jr, Elkayam U, Barbagelata A, Ghali JK, Benza RL, McGrew FA, Klapholz M, et al; Acute and Chronic Therapeutic Impact of a Vasopressin Antagonist in Congestive Heart Failure (ACTIV in CHF) Investigators: Effects of tolvaptan, a vasopressin antagonist, in patients hospitalized with worsening heart failure: A randomized controlled trial. JAMA 291: 1963-1971, 2004.

23. Gheorghiade M, Konstam MA, Burnett JC Jr, Grinfeld L, Maggioni AP, Swedberg K, Udelson JE, Zannad F, Cook T, Ouyang $\mathrm{J}$, et al; Efficacy of Vasopressin Antagonism in Heart Failure Outcome Study With Tolvaptan (EVEREST) Investigators: Short-term clinical effects of tolvaptan, an oral vasopressin antagonist, in patients hospitalized for heart failure: The EVEREST Clinical Status Trials. JAMA 297: 1332-1343, 2007.

24. Slight RD, Demosthenous N, Nzewi OC, Soliman AR, McClelland DBL and Mankad PS: The effect of gain in total body water on haemoglobin concentration and body weight following cardiac surgery. Heart Lung Circ 15: 256-260, 2006.

25. Bellos I, Iliopoulos DC and Perrea DN: The Role of Tolvaptan Administration After Cardiac Surgery: A Meta-Analysis. J Cardiothorac Vasc Anesth 33: 2170-2179, 2019.

26. Crestanello JA, Phillips G, Firstenberg MS, Sai-Sudhakar C, Sirak J, Higgins R and Abraham WT: Postoperative hyponatremia predicts an increase in mortality and in-hospital complications after cardiac surgery. J Am Coll Surg 216: 1135-43, 1143.e1, 2013.

27. Matsuzaki M, Hori M, Izumi T and Fukunami M; Tolvaptan Investigators: Efficacy and safety of tolvaptan in heart failure patients with volume overload despite the standard treatment with conventional diuretics: A phase III, randomized, double-blind, placebo-controlled study (QUEST study). Cardiovasc Drugs Ther 25 (Suppl 1): S33-S45, 2011.

28. Peretto G, Durante A, Limite LR and Cianflone D: Postoperative arrhythmias after cardiac surgery: Incidence, risk factors, and therapeutic management. Cardiol Res Pract 2014: 615987, 2014.

29. Lazzarini V, Bettari L, Bugatti S, Carubelli V, Lombardi C, Metra $\mathrm{M}$ and Dei Cas L: Can we prevent or treat renal dysfunction in acute heart failure? Heart Fail Rev 17: 291-303, 2012.

30. Tominaga N, Kida K, Matsumoto N, Akashi YJ, Miyake F, Kimura K and Shibagaki Y: Safety of add-on tolvaptan in patients with furosemide-resistant congestive heart failure complicated by advanced chronic kidney disease: A sub-analysis of a pharmacokinetics/ pharmacodynamics study. Clin Nephrol 84: 29-38, 2015.

31. Shirakabe A, Hata N, Yamamoto M, Kobayashi N, Shinada T, Tomita K, Tsurumi M, Matsushita M, Okazaki H, Yamamoto Y, et al: Immediate administration of tolvaptan prevents the exacerbation of acute kidney injury and improves the mid-term prognosis of patients with severely decompensated acute heart failure. Circ J 78: 911-921, 2014.

This work is licensed under a Creative Commons Attribution-NonCommercial-NoDerivatives 4.0 International (CC BY-NC-ND 4.0) License. 